\title{
Caractéristiques de la ponctuation dans un corpus de lettres écrites en français par des Finlandais aux $\mathrm{XVIII}^{\mathrm{e}}$ et $\mathrm{XIX}^{\mathrm{e}}$ siècles
}

\author{
Juhani HÄRMÄ \\ University of Helsinki \\ juhani.harma@helsinki.fi \\ https://orcid.org/0000-0002-2969-8583
}

\section{Resumen}

El corpus seleccionado para este estudio está constituido por la correspondencia mantenida entre cuatro finlandeses de finales del siglo XVIII y principios del XIX. Los autores de esta correspondencia comparten ciertos rasgos: todos eran funcionarios de alto rango, que desempeñaron su labor durante un importante periodo de transición en la historia de Finlandia, pertenecían a la nobleza y tenían el sueco como lengua materna; mantenían su correspondencia en sueco y en francés y mezclaban en ocasiones ambas lenguas. Las cartas en francés no muestran una influencia determinante del sueco, salvo los usos de la puntuación. Me concentraré aquí en la raya, cuyo uso quedó establecido en el siglo XIX en varias lenguas europeas.

Palabras clave: correspondencia, puntuación, sociolingüística histórica, contacto de lenguas, francés.

\section{Résumé}

Le corpus de cet article est constitué de correspondances provenant de quatre épistoliers finlandais de la fin du XVIII ${ }^{e}$ et du début du XIX ${ }^{\mathrm{e}}$ siècle. Ces auteurs avaient en commun le fait d'être de hauts fonctionnaires, de travailler durant une période charnière de l'histoire de la Finlande, d'appartenir à la noblesse et d'avoir le suédois comme langue maternelle. Ils s'écrivaient des lettres en suédois et en français, parfois en mélangeant les deux langues. Les lettres écrites en français ne présentent pas d'influence importante du suédois, sauf dans le domaine de la ponctuation. Je me concentrerai sur le rôle du tiret, dont l'usage se stabilise au XIX ${ }^{e}$ siècle dans différentes langues européennes.

Mots-clés : correspondance, ponctuation, sociolinguistique historique, contact de langues, français.

\footnotetext{
*Artículo recibido el 15/09/2020, aceptado el 30/03/2021.
} 


\begin{abstract}
The corpus compiled for the article consists of letters written by four Finnish correspondents active at the end of the eighteenth and the beginning of the nineteenth century. These had several features in common: all were high-ranking officials, working in an important transitional period in Finnish history. They belonged to the nobility and their mother tongue was Swedish. They corresponded with each other in Swedish and in French, sometimes mixing the two languages. The letters written in French do not reflect an important influence from Swedish, except as to punctuation. I concentrate here on the dash, which became established in the nineteenth century in various European languages.
\end{abstract}

Keywords: correspondence, punctuation, historical sociolinguistics, language contact, French.

\title{
1. Introduction
}

Depuis un certain temps, je m'intéresse à des correspondances finlandaises écrites en français et en suédois, qui datent de la fin du XVIII ${ }^{\mathrm{e}}$ siècle et de la première moitié du XIX ${ }^{e}$. En plus de leur intérêt linguistique, les lettres présentent un grand intérêt historique, non-linguistique, comme chroniques de leur époque et comme commentaires des événements européens qui leur sont contemporains. Jusqu'à récemment, ces correspondances n'avaient été étudiées que par des historiens connaissant le suédois et le français. Ces matériaux assez vastes contribuent aussi à placer la Finlande sur une carte de la francophonie européenne ; ce ne sont pas les seuls documents écrits en français par des Finlandais, ni les plus anciens, mais ce sont probablement les plus importants.

Ce qui fait l'intérêt des lettres écrites en français, c'est qu'elles étaient adressées à des compatriotes avec qui les auteurs échangeaient aussi des lettres en suédois. Les lettres étaient donc écrites tantôt en suédois, tantôt en français, sans que les raisons de ces transitions soient évidentes. Les deux langues peuvent alterner aussi dans une même lettre ( $c f$. infra au sujet d'Armfelt, $\$ 4.3$ ). Cette alternance codique est une caractéristique typique, en réalité incontournable, de ces correspondances, et à mon avis la plus intéressante. Elle imprègne les différents domaines que l'on peut choisir comme objet d'étude, qu'il s'agisse du style des épistoliers, des stratégies interactionnelles ou des stratégies de politesse mises en œuvre. Ces correspondances offrent cependant des matériaux également pour des études qui peuvent a priori sembler moins évidentes ou moins intéressantes. C'est le cas des signes de ponctuation, dont l'étude pourrait permettre de découvrir et d'explorer un diasystème rassemblant des scripteurs qui ont plusieurs caractéristiques en commun, tels que les épistoliers dont il sera question ici.

\section{Arrière-plan historique}

Les lettres de mon corpus ont toutes comme auteurs quatre fonctionnaires finlandais, tous issus de la noblesse finlandaise : Gustaf Mauritz Armfelt (1757-1814), 
Johan Albrecht Ehrenström (1762-1847), Robert Henrik Rehbinder (1777-1841) et Carl Johan Walleen (1781-1867). Leurs noms sont inconnus en dehors de la Finlande et de la Suède, même si Armfelt a joué un rôle de second plan dans l'histoire européenne. Ils sont tous nés durant la deuxième moitié du XVIII ${ }^{e}$ siècle, et leurs correspondances proviennent en partie de la fin du XVIII ${ }^{e}$ siècle, mais surtout de la première moitié du XIX'. Il s'agit d'une période charnière dans l'histoire de la Finlande, qui jusque-là faisait partie du royaume de Suède depuis le XII ${ }^{\mathrm{e}}$ siècle. Dans les remous des guerres napoléoniennes, elle est toutefois passée à la Russie en 1809, après une brève guerre entre la Russie et la Suède. Les quatre épistoliers étaient d'abord des sujets du roi de Suède, mais ont ensuite juré fidélité et obéissance à l'Empereur de Russie, Alexandre $\mathrm{I}^{\text {er }}$, et sont devenus, à des titres différents, des fonctionnaires au service de Sa Majesté Impériale.

Le fait que ces quatre épistoliers soient tous des nobles est sans doute en partie une coïncidence, mais pas totalement. Ils ont tous occupé des fonctions importantes, certainement grâce à leur talent et leur compétence, mais sans doute aussi grâce à leurs origines. Ils n'avaient pas tous la même fortune ni la même éducation, mais ils ont appris le français dans leur enfance, soit avec des précepteurs, français ou non, soit avec des membres de leur famille ou de leur entourage. Leur langue maternelle était en principe le suédois, mais le concept de langue maternelle était à l'époque plus flou qu'actuellement. Les épistoliers pouvaient utiliser le français au sein de leur famille ou avec des amis. Les lettres démontrent que leur français n'est pas parfait, mais on peut constater qu'ils faisaient des "fautes" également quand ils écrivaient le suédois. De toute façon, une espèce de connivence s'établit entre eux, parce qu'ils sont capables de communiquer en français, mais le fait qu'ils étaient tous nobles les unissait également.

Le français avait commencé à gagner de l'importance en Suède au XVII ${ }^{\mathrm{e}}$ siècle et sa position s'est consolidée durant la deuxième moitié du XVIII siècle, surtout pendant le règne du roi francophile Gustave III (né en 1746, il régna de 1770 à 1792) ${ }^{1}$. Il faut noter que le finnois, langue parlée par la majorité de la population, ne joue aucun rôle dans ce paysage sociolinguistique, puisqu'il n'était parlé que par les couches inférieures de la population et que les classes supérieures n'employaient cette langue que pour parler avec les domestiques ou ne la parlaient pas du tout ( $c f$., à ce propos, l'excellent survol de Saari, 2012). En ce qui concerne le russe, langue des dirigeants à partir de 1809 jusqu'à l'indépendance du pays en 1917, les Finlandais ne l'employaient guère et communiquaient en français avec le Gouverneur général, représentant de l'empereur en Finlande, et son état-major, ainsi qu'avec la cour de Saint-Pétersbourg.

${ }^{1}$ Sur le français en Suède et en Finlande, $c f$. Pettersson (2005 : 126-128) ; Lindgren \& Lindgren (2005: 256-260); Rettig (2006: 1807-1808); Wolf (2008: 3327-3329); Östman \& Östman (2008); Östman (2012). 
Les fonctionnaires finlandais en poste à Saint-Pétersbourg² (Walleen, Rehbinder, Armfelt) utilisent le français comme lingua franca et se débrouillent sans connaître le russe. Des documents conservés aux Archives nationales de Finlande démontrent qu'un grand nombre de documents officiels rédigés en Finlande pendant le XIX ${ }^{\mathrm{e}}$ siècle, sous le règne russe, étaient en français, même si cette langue n'avait évidemment aucune position officielle en Finlande et qu'elle n'était parlée que par un pourcentage réduit de la population.

La Finlande a donc d'abord subi, à la fin du XVIII ${ }^{e}$ siècle, l'influence de la cour francophone du roi Gustave de Suède, et ensuite c'est la cour francophone de SaintPétersbourg qui a été émulée. Le suédois demeure l'autre langue de l'élite pendant le $\mathrm{XIX}^{\mathrm{e}}$ siècle. Voilà donc une situation sociolinguistique un peu complexe, où des langues ignorées par la majeure partie des Finlandais servaient de langues véhiculaires au sein d'une petite partie de la population. Il s'agit d'une situation particulière de contact de langues où l'une des langues, le français, n'a aucun rapport direct (géographique ou historique) avec le territoire où elle est parlée, et les deux langues (français et suédois) sont en fait minoritaires sur ce territoire. Le rôle du français dans ce contexte est évidemment dû à son prestige européen.

\section{Corpus}

Cet arrière-plan historique et sociolinguistique me semble nécessaire dans ce contexte (pour plus d'informations sur un tel contexte, $c f$. Härmä, 2013, 2015, 2020). Le corpus dont je me sers est difficile à cerner. Il est constitué des correspondances des quatre épistoliers qui ont été présentés ci-dessus, et il est composé de lettres qu'ils se sont envoyées les uns aux autres, mais aussi à d'autres correspondants, surtout dans le cas d'Armfelt. Les lettres sont conservées aux Archives Nationales de Finlande à Helsinki, et elles ont récemment été numérisées. Le nombre total des lettres écrites par chaque correspondant n'a pas été indiqué par les Archives, et il semble que lors de la numérisation, personne n'ait compté les lettres. On m'a cependant signalé que la correspondance française conservée d'Ehrenström est constituée de plus d'un millier de lettres. Cela doit être le cas également des lettres d'Armfelt, comme on peut le déduire du nombre des photographies des lettres disponibles sur internet : en tout, plusieurs milliers.

\footnotetext{
${ }^{2}$ Ehrenström travaillait à Helsinki, la nouvelle capitale du Grand-Duché de Finlande, dont il assura la reconstruction et la rénovation à partir de 1812. Ses collègues Walleen, Rehbinder et Armfelt étaient en poste à Saint-Pétersbourg au service de l'Empereur pendant les premières décennies du XIX ${ }^{\mathrm{e}}$ siècle, assurant des fonctions différentes, mais œuvrant néanmoins pour la Finlande. Les correspondances dont il est question ici sont basées sur les échanges qu'Ehrenström entretient avec Rehbinder d'une part et Walleen de l'autre. Les correspondances d'Armfelt étudiées ici sont en général plus anciennes, datant de sa période suédoise précédant l'assassinat de Gustave III en 1792 (cf. \$4.3.).
} 
Les lettres d'Armfelt sont peut-être les plus intéressantes, mais elles ont été mal cataloguées, les reproductions sont souvent de mauvaise qualité et son écriture est malheureusement difficile à lire 3 . D'une manière générale, les correspondances s'échelonnent sur une période de 55 ans environ (les deux dernières décennies du $\mathrm{XVIII}^{\mathrm{e}}$ siècle et les trois ou quatre premières du XIX ${ }^{\mathrm{e}}$ siècle). Seul Ehrenström, mort à 84 ans, a eu l'occasion d'écrire des lettres pendant plus de 50 ans. Il y a eu des changements dans la langue pendant cette période de deux générations (qu'il s'agisse du suédois ou du français, tel qu'il est utilisé par les épistoliers), et il serait possible, en théorie, de baser une étude diachronique sur ce corpus. Ces changements peuvent être notamment dus au fait que le début du XIX ${ }^{\mathrm{e}}$ siècle, période charnière du point de vue historique, signifie aussi le début d'écarts langagiers pour le suédois de Finlande par rapport au suédois de Suède, ce qui peut avoir un effet indirect sur le français utilisé par ces suédophones (Ahlbäck, 1971 : 29). En Suède, l'utilisation des mots étrangers ou français a été restreinte par un décret au début du $\mathrm{XIX}^{\mathrm{e}}$ siècle, mais cela ne concernait plus la Finlande, détachée à cette époque de la Suède. De toute façon, je ne tenterai pas une approche diachronique ici (même si ces lettres se trouvent dans la diachronie du français), pas plus qu'une approche quantitative. Cette étude se place cependant dans un cadre de sociolinguistique historique.

Il est légitime de s'interroger sur le statut ou l'intérêt éventuel d'un tel corpus assez volumineux, composé de correspondances en français et provenant de quatre personnes différentes dont aucune n'a le français comme langue maternelle officielle ${ }^{4}$. Quelle importance un tel corpus peut-il avoir pour la francophonie ou pour des recherches portant sur le français des siècles passés? Il est évident qu'une étude diachronique portant sur ces lettres écrites pendant une cinquantaine d'années (entre la fin du XVIII et le début du XIX ${ }^{\mathrm{e}}$ siècle) ne révèlerait rien d'intéressant en ce qui concerne les changements linguistiques de la langue française.

Les contacts de langue français-suédois constituent quant à eux une question intéressante, mais ne nous concernent pas directement ici, même si le recours à la langue suédoise est inévitable jusqu'à un certain degré. Le français des quatre épistoliers ne représente pas une variété uniforme de langue, mais présente quand même un certain nombre de caractéristiques qui valent à mon sens la peine d'être étudiées ( $c f$.

\footnotetext{
${ }^{3}$ Notons à ce propos que les fautes de français relativement nombreuses des exemples donnés ici sont dues aux auteurs eux-mêmes (à l'exception de quelques erreurs éventuelles survenues durant le travail de copie). Les soulignements dans les exemples proviennent des auteurs, tandis que les passages entre crochets, donnant des clarifications, sont dus à moi.

${ }^{4}$ Les Archives Nationales de Finlande possèdent aussi des correspondances d'autres personnes ou de réseaux de correspondants, dont l'arrière-plan ou le contexte langagier est grosso modo le même que celui d'Ehrenström et de ses collègues et amis. Il y a, par exemple, un ensemble de lettres écrites durant la deuxième moitié du XIXe siècle par Aurora Karamzin, aristocrate finlandaise ayant passé quelques dizaines d'années à la cour de Saint-Pétersbourg, et par son cercle de proches et d'amis.
} 
Härmä, 2013, 2015, 2020). Le français tel qu'il a été écrit en Finlande depuis le XVII siècle a été largement ignoré et éclipsé par le français de Suède, pour des raisons évidentes. Cette variante "finlandaise " $\mathrm{du}$ français vaut cependant la peine d'être reconnue comme faisant partie intégrante de la francophonie européenne des pays où le français n'est pas une langue majoritaire.

On peut également se demander comment définir ou classer les lettres de ce corpus imposant. Les correspondances d'Ehrenström, de Rehbinder et de Walleen pourraient être qualifiées de "semi-officielles". Ce sont des lettres de fonctionnaires, presque toujours très sérieuses et pondérées, qui discutent pour la majeure partie de questions administratives ou politiques ${ }^{5}$. Cependant, la vie personnelle des épistoliers apparaît en passant dans presque chaque lettre, surtout lorsqu'ils parlent de leurs problèmes de santé ( $c f$. citations 1, 6-8) ou, par exemple, lorsqu'il est question d'achats à faire à Saint-Pétersbourg ( $c f$. citations 6, 19 et 22). On peut se poser la question de savoir si les auteurs ont pensé à la postérité en écrivant ces lettres, mais la discussion de cette question demanderait un développement un peu trop long; Armfelt est un cas différent, parce qu'une grande partie de sa correspondance est privée ( $c f$. infra, $\$ 4.3$ ), et ce n'était pas un fonctionnaire au même titre que les trois autres.

\section{Ponctuation}

\subsection{Généralités}

Il n'y a évidemment pas lieu de présenter ici un historique de la ponctuation en suédois. Constatons cependant que les règles de la ponctuation phrastique de cette langue étaient différentes de celles du français. Jusque vers le milieu du $\mathrm{XX}^{\mathrm{e}}$ siècle, le suédois suivait une ponctuation démarcative, où il convenait de distinguer ou de séparer chaque proposition avec un signe (Gaeta, 2008 ; Strömquist, 2013 ; Dahl, 2016 : 41). Par exemple, la virgule devant la conjonction équivalant au complémenteur français que était obligatoire, ce qui n'est plus le $\operatorname{cas}^{6}$. L'exemple suivant, tiré d'une lettre envoyée en 1819 par Ehrenström à Rehbinder, illustre cette pratique :

(1) La derniere lettre dont Vous m'avez honoré, Monsieur le Baron, du $10 / 22^{7}$, me comunique l'agréable nouvelle, que Votre Santé commence à se

\footnotetext{
${ }^{5}$ Ehrenström et Walleen s'écrivent en 1814 et 1815 de longues lettres contre Napoléon, la France et les Français. Ces lettres, où les auteurs ne réussissent guère à garder leur calme, représentent le " genre épistolaire politique » et sont presque des essais politiques.

${ }^{6}$ Cf. la situation en danois, langue apparentée de près au suédois : Korzen (2017 : 334-335) présente la distinction entre la virgule logico-sémantique ("virgule marquant la pause ») et la virgule grammaticale ( virgule traditionnelle »). Depuis 1996 a été adoptée une nouvelle virgule unique, marquant les pauses naturelles.

${ }^{7}$ Cette indication de temps renvoyant à deux dates en mai 1819 est basée sur la différence entre les calendriers julien (le 10), toujours utilisé en Russie à l'époque, et grégorien (le 22), utilisé en Finlande (et en Suède). L'utilisation des deux dates est courante chez ceux qui sont en Russie et envoient des lettres en Finlande, et à l'inverse.
} 
retablir parfaitement. Vous n'auriez pas pû m'en donner une, qui m'eut fait plus de plaisir (Ehrenström à Rehbinder, 1819).

Cependant, les épistoliers finlandais n'observent nullement cette règle d'une manière systématique, et leur ponctuation dans les lettres écrites en français est proche de la ponctuation française. Voici quelques exemples variés qui illustrent différents emplois de la ponctuation et ne correspondent pas vraiment aux règles ni aux usages de l'une ni de l'autre langue ${ }^{8}$ :

(2) Il est très probable que la nouvelle, déjà inserée dans les gazettes Allemandes, sur la résolution prise par l'Empereur, de défendre à ses sujets de fréquenter les Universités Allemandes, se confirme (Ehrenström à Rehbinder, 1819).

(3) La Note Russe ci-jointe, a été donné par le marchand Russe Sinébrukoff, pour indiquer où trouver le Négociant Uschakoff qui mieux que tout autre, pourra trouver une occasion sûre pour nous faire parvenir les parquets (Ehrenström à Rehbinder, 1819).

(4) Ce qu'on en dit, ne peut pas être un Conte en l'air, depuis que le Général d'Opperman en a fait la matiere d'une lettre officielle, dans laquelle cependant le nom du Prince n'est pas articulé (Ehrenström à Rehbinder, 1819).

(5) Je mande cela, uniquement pour ajouter, que ce n'est pas par moi qu'on l'a sû (Ehrenström à Rehbinder, 1819).

Les signes de ponctuation déjà usuels se retrouvent dans mon corpus de lettres vers 1800 : ainsi, en plus du point et de la virgule, on trouve bien sûr le point d'interrogation, comme le point-virgule, le point d'exclamation, les guillemets et les deux points. Par contre, l'utilisation des parenthèses est peu courante, et certains épistoliers semblent ne pas les employer du tout ${ }^{9}$. Je n'ai pas trouvé d'exemples des points de suspension dans mon corpus; en fait, c'est le tiret qui semble souvent jouer leur rôle. Mes affirmations sur l'absence de tel ou tel phénomène dans la correspondance de tel auteur sont sujettes à caution, puisqu'il n'est pas possible de lire toutes les lettres, soit à cause de leur grande quantité, soit parce que toutes les lettres ne nous ont pas été conservées. L'utilisation de la virgule, en particulier, défiant parfois toute logique, invite à faire des comparaisons avec l'utilisation désinvolte, pour ne pas dire négligente, des accents dans les quatre épistoliers ${ }^{10}$. On en voit des exemples ci-dessus, et d'autres exemples suivront.

\footnotetext{
8 Tous ces exemples proviennent de lettres écrites par Ehrenström à Rehbinder.

${ }^{9}$ Rehbinder s'en sert cependant.

${ }^{10}$ L'emploi des accents chez Rehbinder suit cependant une certaine logique, quoique pas tout à fait systématique. En général, il se sert de l'accent aigu en fin de mot, mais omet facilement les accents qui précèdent ; par exemple, faché, oté, cotés, gouté ; cf., aussi, espece, veritable, detail, amerement, etc. D'autre part, il arrive également qu'il ajoute des accents là où il n'en faut pas, peut-être par hypercorrection : des
} 
Je présenterai à la fin de cet article quelques remarques d'ensemble sur les caractéristiques de l'écriture des quatre épistoliers. Une étude comparative serait cependant trop longue et ne présenterait pas suffisamment d'intérêt du point de vue de la langue française des siècles passés. Il faut encore souligner que les épistoliers ne semblent pas suivre de règles précises dans l'emploi qu'ils font des signes de ponctuation.

Je vais me concentrer sur le tiret, puisque ce signe présente des emplois particuliers qui semblent n'appartenir qu'au suédois du XIX ${ }^{e}$ siècle, et, par là, au français écrit par les suédophones à cette époque.

\subsection{Le tiret chez Ehrenström, Rehbinder et Walleen}

Selon Catach (1980 : 17), le tiret fait partie des signes d'énonciation, ce qui est déjà apparent dans les correspondances étudiées ici. Il assure des fonctions relevant de l'organisation syntaxique, mais aussi relevant de l'oralité. En simplifiant un peu, on peut dire que les emplois principaux actuels du tiret - ceux qui nous concernent ici sont au nombre de deux : d'une part, (a) le tiret peut indiquer un ajout, un complément d'information, en fin de phrase ou bien au milieu (dans ce dernier cas, il sera accompagné d'un deuxième tiret), et d'autre part, (b) il peut mettre en valeur, en relief, un mot ou un syntagme ${ }^{11}$. (c) Le tiret s'emploie bien sûr également dans le dialogue pour indiquer la prise de parole, ainsi que (d) comme symbole dans les indications de temps $(12-14$ h.) ou de distance (le train Madrid - Salamanque). Ces deux derniers emplois ne nous concernent pas $\mathrm{ici}^{12}$.

Le tiret est mentionné pour la première fois dans la littérature de référence en suédois en 1813 ; le terme suédois de tankstreck (qui est un calque de l'allemand Gedankenstrich et dont une traduction littérale en français pourrait être " trait/tiret de réflexion ») est utilisé dans un manuel de suédois paru cette année-là ( $c f$. Strömquist, 2013 : 60). Le tiret n'est apparemment utilisé en suédois qu'à partir du XVIII ${ }^{\mathrm{e}}$ siècle (cf. Dahl, 2016 : 34ss.). Curieusement, il existe très peu d'études ou de données d'ordre diachronique sur le tiret (dit "de réflexion») en suédois, et cela vaut pour la

vôeux bien ardents. Sans présenter de statistiques, on peut toutefois assurer qu'il omet la majorité des accents.

${ }^{11}$ Selon Barsi (2008 : 283-284), le tiret (a) relance le discours, (b) introduit un changement de point de vue ou (c) indique l'irruption d'une autre voix narrative (elle donne des exemples des Mémoires d'outretombe, provenant donc de la première moitié du XIX ${ }^{e}$ siècle). Barsi (2008: 284) présente aussi des exemples d'une pièce de Musset, où, d'après elle, le tiret a une valeur énonciative.

${ }^{12}$ Ehrenström utilise le tiret dans des mots composés au lieu du trait d'union, emploi similaire aux indications de distance ou de temps. L'emploi de ces signes ne s'était donc pas encore stabilisé à la fin du XVIII s. ou au début du XIX ${ }^{\mathrm{e}}$ s. (par exemple, människo - ödande 'homme - dévorant' [" qui consume un homme »]). Cette classification relativement simple n'est évidemment pas toujours suivie ; Drillon, par exemple, propose plusieurs groupes différents. Il constate également que le tiret «conclut ou interrompt» (1991 : 338). Sur le tiret en général, cf. Drillon (1991 : 329-340) ; Catach (1994: 75 76) ; Bredel (2011 : 43ss.) ; Holmes \& Hinchliffe (2013 : 687); Dahl (2016 : passim). 
ponctuation suédoise en général ${ }^{13}$. Si la ponctuation des lettres écrites en français que nous étudions semble en général avoir subi une influence de la part du français, par exemple pour la virgule, il semble plus que probable, pour le tiret, que l'influence vienne du suédois.

Le tiret apparaît dans mon corpus dès la fin du XVIII ${ }^{e}$ siècle, au moins dans les lettres de jeunesse d'Armfelt (et chez lui, dès les années 1780). La codification de la langue suédoise n'est pas encore terminée à l'époque ( $c f$. Ahlbäck, 1971 ; Pettersson, 2005) et, de toute façon, les connaissances grammaticales des épistoliers ne sont pas approfondies. Même s'ils connaissent tous le français nettement mieux que plusieurs de nos contemporains, leur culture générale varie beaucoup ${ }^{14}$. Ehrenström et Armfelt surtout, que seules cinq années séparaient, étaient des hommes du XVIII siècle, mais les autres avaient aussi été scolarisés avant le début du XIX ${ }^{e}$ siècle, et leur français s'en ressent.

Ce qu'il y a de particulier dans la majorité des tirets de mon corpus, c'est, d'une part, qu'ils se placent en bas de ligne et non dans la position à laquelle nous sommes habitués (milieu de la ligne) ${ }^{15}$. D'autre part, leurs fonctions ne semblent souvent pas correspondre à l'usage actuel, si on pense à des langues comme le français et les autres langues romanes ou encore comme le suédois, l'anglais, le finnois, etc. Les emplois semblent par ailleurs varier selon les épistoliers. Les fonctionnaires Rehbinder et Ehrenström s'en tiennent à un usage " modéré », peut-être dû à leur statut professionnel, tandis qu'Armfelt s'en sert souvent d'une manière débridée; dans son cas, il est évident que l'utilisation du tiret varie selon les destinataires de ses lettres et évolue aussi avec l'âge (v. infra, $\$ 4.3)^{16}$.

\footnotetext{
${ }^{13}$ Communications personnelles de deux professeures de langues scandinaves de l'Université de Helsinki, Mirja Saari et Hanna Lehti-Eklund.

${ }^{14}$ Rehbinder et Walleen avaient un diplôme universitaire de droit, tandis qu'Ehrenström était autodidacte, n'ayant pu être scolarisé par son père pour des raisons économiques. Malgré cela, il apprit plusieurs langues étrangères à part le français.

${ }^{15}$ La position du tiret évolue au cours du XIXe siècle. Eliel Kilpelä, rédacteur des Euvres complètes numérisées de l'écrivain finlandais de langue suédoise Zacharias Topelius (1818-1898), a attiré mon attention sur le fait qu'avant le milieu du XIX siècle, le tiret commence à se déplacer de haut en bas et de bas en haut et à se stabiliser peu à peu dans sa position actuelle dans la production de l'écrivain. On peut supposer que cela concerne aussi la production d'autres écrivains contemporains, mais il semble que personne n'ait étudié la question de la place du tiret et des changements portant sur celle-ci.

${ }^{16} \mathrm{Il}$ serait téméraire de prétendre que l'emploi du tiret corrèle avec la fonction et la carrière de tel épistolier, mais les correspondances d'Ehrenström, de Rehbinder et de Walleen dont nous disposons consistent dans des lettres semi-officielles, où il y a peu de place pour la fantaisie, si ce n'est pour un peu d'humour et d'ironie. Armfelt a eu une vie d'aventurier et a porté plusieurs casquettes; il était officier, diplomate, homme politique, fonctionnaire, homme de lettres et de thêâtre, membre de l'Académie suédoise, etc.
} 
Ce qui surprend, c'est l'emploi du tiret en apparence aléatoire et d'une fréquence très variable. Il peut apparaître aussi bien dans les lettres en suédois que les lettres en français (je ne donnerai ici que des exemples en français) :

(6) Les catheter coutent ensemble 25 Roubles. Quant aux livres _ j'ai perdu la note mais les prix en sont conformes aux catalogues. Plusieurs des livres que Vous avez demandé ne se trouvaient plus chez Kennier ? (Rehbinder à Ehrenström, 1819).

(7) Ma santé va beaucoup mieux les forces commencent à revenir mais la digestion est toujours lente (Rehbinder à Ehrenström, 1819).

(8) La derniere Poste de St. Petersbourg ne m'apporta aucune information sur l'état de Votre Santé, Monsieur le Baron. C'est cependant un article du plus haut interêt pour moi. _ La mienne va mieux (Ehrenström à Rehbinder, 1819).

Je présente maintenant une classification possible, même si d'autres sont également envisageables :

(a) Commençons par les cas qui sont identiques à la pratique moderne ou qui s'en rapprochent de manière évidente, à savoir ceux qui introduisent un ajout ou un complément d'information.

(9) Tous çeux qui m’ont parlé de lui [un ami qui agonise], _ et ils sont en grand nombre, _ ont été d'accord sur çe point, et je suis bien sûr que ses calomniateurs même, s'il en a d'autres que le seul homme, qui dans sa démençe se plait à faire la guerre à tous les honnêtes gens d'un mérite plus eminent que le sien _ que ses calomniateurs même, dis-je, seront forçés de se taire devant la voix publique [...] $]^{17}$ (Walleen à Ehrenström, 1815).

(10) Heureux celui qui peut se retenir dans un coin, avant cette époque et je tache chaque jour de me famillariser avec l'horrible ideé de me séparer de propos delibéré d'un souverain et d'un Bienfaiteur quej'adore_c.a.d. me separer de sa personne, mais jamais de vivre sous son Gouvernement (Rehbinder à Ehrenström, 1819).

(11) Les politiques de la Capitale pretendent que nous aurons bientot la guerre __ qu'une alliance s'est formé contre nous, composée de l'Autriche, la Bavière, l'Angleterre et la Suède [...] (Rehbinder à Ehrenström, 1819).

L'exemple (9) est proche des usages contemporains. On pourrait imaginer, à la place des deux premiers tirets qui encadrent l'ajout, également des parenthèses. Le troisième tiret, qui ne se rattache pas directement aux deux précédents, permet de structurer la phrase, assez complexe, qui continue encore sur plusieurs lignes après la fin de la citation (Walleen a l'habitude des phrases longues et sinueuses, et en général, il réussit

${ }^{17}$ Cet exemple démontre bien une particularité pléonastique des habitudes orthographiques de Walleen, qui met en général une cédille sous les $c$ qui se prononcent comme un $s$. On constate le même phénomène dans certaines lettres d'Ehrenström (v. ex. 16). 
à les construire de manière à les rendre correctes et compréhensibles). Il s'agit d'un décrochage énonciatif ( $c f$. Llamas-Pombo, 2017), où l'épistolier en quelque sorte affirme sa présence et se reprend au milieu d'une phrase à la structure complexe. Les exemples (10) et (11) contiennent aussi un ajout ou une explication supplémentaire après le tiret, qui pourrait être remplacé par un point-virgule.

(b) Assez souvent, on peut avoir l'impression que le tiret reflète effectivement un moment de réflexion, comme son nom allemand ou suédois l'indique (cf. supra). On pourrait bien sûr objecter qu'il s'agit là d'une impression tout à fait subjective : comment pouvons-nous savoir que l'épistolier réfléchit au moment où il trace un tiret ? Considérons toutefois les exemples suivants :

(12) Selon lui [= une "gazette »], Bonaparte n'étoit entré en Françe qu’avec une poignée de soldats, personne ne s'étoit réuni à lui; son entreprise n'étoit que ridicule, folle, extravagante, effet de son désespoir etc. _ Et tandis qu'on lit à Paris toutes çes belles çhoses, Bonaparte avec sa poignée d'hommes, insulte déjà, dans la seconde ville du Royaume, le Gouvernement légitime de la Françe, des corps entiers de soldats désertent pour se rallier sous les drapeaux; un ministre du Roi est de ses satellites, et son entreprise ridicule est devenue fort sérieuse (Walleen à Ehrenström, 1815).

(13) Je n'ai rien entendu parler de cette scene entre deux professeurs d'Abo ${ }^{18}$. __'en suis bien faché et je me figure la vive joie que cela doit exciter parmi ceux qui ont adopté le principe de denigrer tout ce qui tient a notre administration actuelle et surtout d'humilier et ridiculiser la seule academie que nous ayons et qui par preference a eu le tort d'elever les ignorans et les imbecilles qui sont revetus de nos premieres places de magistrature (Rehbinder à Ehrenström, 1819).

(14) Le vieux Sprengtporten est mort et enterré. Il avait en soi les elemens d'un grand homme__ un coin de sa tête etait un chef d'oeuvre mais il parait comme si le reste n'avait pas été achevé par le même maitre (Rehbinder à Ehrenström, 1819).

(15) J'ai eté bien mal _ mais je me trouve assez retabli pour penser de faire ma premiere sortie en voiture aujourd'hui. Je n'ai pu travailler chez l'Empereur jeudi passé (Rehbinder à Ehrenström, 1819).

C'est surtout Rehbinder qui affectionne dans ses lettres ces ajouts ou ces compléments d'information, qui semblent être le fruit d'une réflexion plus poussée. On pourrait dire que l'épistolier donne l'impression de poser sa plume pour un instant avant de continuer et pour reprendre son "souffle " (cf. Catach, 1980:23; Serça, 2004 : 15). Le tiret pourrait être remplacé par d'autres signes de ponctuation, ou être simplement omis comme dans l'exemple (12), celui de Walleen. Le topique ne change

${ }^{18}$ Renvoi à une lettre précédente d'Ehrenström, où celui-ci rapporte une altercation entre deux professeurs de l'Académie (à savoir l'Université) de Turku/Åbo, qui a fait scandale. 
pas (cf. ci-dessous groupe (c), " ordre du jour »), mais le point de vue ou la perspective peut changer. De toute façon, le tiret n'indique pas un ajout parenthétique ou une nouvelle information mise en valeur ; il indique plutôt un développement de ce qui a été dit précédemment. On pourra le considérer comme une sorte de marqueur d'énonciation, de décrochage énonciatif : une indication d'une nouvelle réflexion de l'épistolier au sujet du topique qu'il a discuté et qu'il continue de discuter.

(c) Dans certaines lettres, le tiret se retrouve plusieurs fois, à des intervalles plus ou moins réguliers, sur une page ou deux. Il marque dans ce cas-là assez nettement les points d'un « ordre du jour» :

(16) Monsieur!

Votre lettre, Monsieur, du 17, est arrivée en deux jours, le 19. Elle m'en annonce une autre plus longue, envoyée avec Mr. de Willebrand, Conseiller d'Etat actuel. Je ne puis pas esperer à la reçevoir que vers la fin de la semaine prochaine, car un homme de mon âge qui se marie, a besoin de voyager lentement, à petites journées, afin de menager ses forçes. __ Mille graçes pour la peine que Vous avez bien voulû Vous donner à l'égard de mes commissions. Les nouvelles de la défaite de Murat, paroissant se confirmer de toute part, au grand déplaisir d'une partie du public de Stockholm, qui avoit déjà mis en circulation le bruit qu'il avoit complettement battu les Autrichiens, que toute l'Italie s'étoit levée en sa faveur, qu'on avoit fait main basse sur les Allemands, etc. (Ehrenström à Walleen, 1815).

(17) On soutient avec ardeur le principe, que la Nation Françoise, ayant reçu Buonaparte avec enthousiasme, et se préparant à le reélire pour la seconde fois Empereur des François, personne n'a le droit de mettre en doute le sien, constaté si solemnellement. J'avoue que la farce qui va être jouée au Champ de Mai, et dont le resultat n'est pas douteux, est profondement calculée pour inspirer de justes allarmes, parcequ'elle donne un air de légitimité à la nouvelle usurpation du Corse. La Suède ne s'est pas encore prononcée pour la grande cause, mais comme on prétend que le Congrès de Vienne a garanti au Roi de Danemark la possession de la Poméranie Suédoise, que la Cour de Stockholm ne veut pas lui ceder de bon gré, on a envoyé des troupes à Stralsund, sous les ordres du General de Döbeln, qu'on dit être enchanté de cette marque de confiance. ___ Je suis très-inquiet sur le sort du Duc d'Angoulême, parceque je crains toujours qu'il sera la victime de la perfidie de Buonaparte. Nous ne savons pas encore avec certitude qu'il soit à l'abri du danger. Voilà un bel exemple que Mr. de Willebrand nous donne. Pour moi, je suis déjà trop vieux pour le suivre, mais pour Vous, Monsieur, il ne doit pas être perdû. __ Point de reponse encore de Vienne. ___ Que le Tout puissant daigne protéger notre Bon Empereur, et qu'Il puisse réussir à raffermir la tranquillité de l'Europe, pour la destruction complette de l'Hydre revolutionnaire de France !!! ___ Presentez, je Vous prie, Monsieur, mes complimens et respects à Mr. et Madame de Rhebinder [sic], et agréez l'expression de mes 
sentimens d'attachement et d'amitié sincère pour Votre personne (Ehrenström à Walleen, 1815).

Cet emploi s'observe surtout chez Ehrenström, dont l'esprit méthodique semble favoriser les énumérations de questions à discuter et à résoudre. L'emploi des tirets pour structurer une liste n'est cependant pas très courant même dans ses lettres, et, comme chez les autres épistoliers (à l'exception d'Armfelt), les lettres contenant des tirets sont nettement en minorité ; il est même possible de trouver une longue lettre avec un seul tiret. Dans tous les cas, le tiret annonce dans cette catégorie d'emploi un changement de topique, à l'opposé des exemples des groupes précédents. Dans l'exemple (17), un point soulevé par Ehrenström, Point de reponse encore de Vienne (mention d'un sujet traité précédemment par les deux épistoliers) ne consiste qu'en six mots, tandis que d'autres points ont droit à plusieurs lignes.

On peut inclure sous cette rubrique également les changements brusques de topique, comme (18), où l'épistolier tient à marquer (ou peut-être à justifier) le changement soudain de thème.

(18) Les nouvelles sur l'arrivée de l'Empereur sont toujours inçertaines. On en parle beaucoup; mais çe qu'il y a de sûr, c'est que personne n'en sait rien. _ Un bruit s'est répandu içi de la mort du Roi de Françe, sans qu'on y ajoute beaucoup de foi, et je crois qu'en çela on fait fort bien (Walleen à Ehrenström, 1815).

(19) En attendant, je suis laissé, par l'absence du Souverain, dans une situation extrêmement penible et ne sait plus où tourner la tête. Il faut une opiniatreté telle que la mienne, pour rester attaché à la mémoire de mon ami, le Comte d'Aminoff, qui, par legereté, m'a mis dans l'embarras ou je me trouve.

Malheureusement les deux schawls, que j'ai maintenant vû moi-meme, ont été gatés par la pluie, surtout l'un des deux, où il y a des tâches grandes comme une soucoupe. Je crains maintenant que le schawl bleu, que j'attends avec le courier de demain, aura le même sort, si le paquet n'est pas enveloppé de toile cireé. __ Présentez, je vous prie, mes respects à $\mathrm{M}^{\mathrm{e}}$. et $\mathrm{Mad}^{\mathrm{e}}$. de Rhebinder, et ne doutez jamais de la sincerité des sentimens d'estime et d'amitié que je vous porte (Ehrenström à Walleen, 1815).

Dans l'exemple (19), dernier paragraphe d'une lettre, les tirets encadrent un passage cocasse où Ehrenström revient, une fois de plus, sur l'affaire de deux châles ("schawl») de Boukhara qu'il avait commandés à Saint-Pétersbourg pour une dame, jamais nommée, et qui sont arrivés abîmés à Helsinki. Le passage peut faire sourire dans une lettre qui traite par ailleurs de questions administratives, ce qui est normalement le cas dans ces correspondances. (Il est vrai que parmi ces questions, la démission envisagée par Ehrenström est au premier plan, de sorte que la mention des deux châles renchérit sur l'aspect plus personnel de la lettre). 
(d) Le tiret peut indiquer un décrochage énonciatif (Badiou-Monferran, 2017 ; Pétillon, 2004), une sorte de rupture ou de passage d'un niveau ou d'un mode à un autre. Dans les trois exemples ci-dessous, on passe à une sorte d'oralisation ou d'embrayage : l'épistolier (différent dans chaque exemple) s'adresse plus directement à son correspondant après le tiret. Dans (22), qui est un ajout dans la marge de la dernière page de la lettre, Ehrenström présente deux requêtes à Rehbinder (comme il en fait souvent aussi bien à celui-ci qu'à Walleen), en utilisant des stratégies de politesse avec deux propositions conditionnelles de types différents. Le tiret sépare les deux requêtes, peut-être pour créer une distance entre elles et amortir le fait qu'il y en a plus d'une :

(20) [...] toute la Capitale étoit électrifiée en faveur du Roi, tous les députés des deux çhambres avoient juré, les larmes aux yeux, de mourir pour lui, tous les mareçhaux avoient fait le même serment dans leurs adresses à sa Majésté; enfin tout étoit au mieux possible. _. Eh bien! que dites-vous, Monsieur, quand Vous saurez que malgré tout çela Bonaparte est à Paris, toute l'armée à ses ordres, toute la nation soumise de nouveau à sa tyrannie, le Roi en fuite, et le malheur à son comble! (Walleen à Ehrenström, 1815)

(21) Jusqu'ici j'ai eu d'estime pour cet homme faible [le Sieur Etter], mais actuellement je suis faché d'etre obligé d'en rabattre une bonne partie. Cet etat de choses ne durera pas longtemps _ Tout ici entre nous (Rehbinder à Ehrenström, 1819).

(22) Si Vous vouliez bien avoir la bonté de faire acheter pour moi une boïte de 5 livres de tabac, de celui de Charron la Rose. Le General de Reuterskjöld auroit bien la bonté de me l'apporter. __ Si je ne suis pas trop importun, j'ose Vous rappeller le $\mathrm{n}^{\circ} .19$ du Conservateur Impartial pour cette année, qui me manque et que Votre courier pourroit peut-être trouver dans l'Imprimerie (Ehrenström à Rehbinder, 1819).

(e) Plusieurs cas restent sans explication valable, du moins du point de vue de notre époque moderne. Walleen, par exemple, termine souvent ses paragraphes avec un tiret :

(23) Je me borne donc à Vous dire seulement, que de tous les plaisirs qui s'offrent içi à moi, il n'y a aucun qui saurait me dedommager de la perte de çelui que je viens de gouter dans Votre société; qu'il me tarde en conséquence d'en jouir de nouveau; et que ma reconnaissançe de Vos proçédés à mon égard égalera à jamais l'estime profonde que mon coeur Vous a vouée, et que Vous méritez à tant de titres. _ (Walleen à Ehrenström, 1815).

(24) Trop d'humanité envers un homme comme Bonaparte est de l'inhumanité envers tous les autres. On fera sans doute maintenant çette reflexion, mais un peu tard pour tous çeux qui tomberont dans çe dernier combat qu'on aura encore à soutenir, pour débarasser enfin le monde de çe fou enragé, qui tant qu'il existe ne çessera jamais d'en troubler le repos, ou de faire au moins pour 
çela des tentatives, toujours inquiétantes, quand même elles ne seroient pas suivies de succès. _ (Walleen à Ehrenström, 1815).

Dans (23), il s'agit du premier paragraphe de la lettre, tandis que dans (24) on a affaire à un paragraphe qui apparaît en avant-dernière position et qui n'est suivi que par les salutations de rigueur en fin de lettre. Il arrive même que Walleen termine une ligne par un tiret, au beau milieu d'une phrase, comme s'il s'agissait de combler une ligne restée incomplète. Dans l'exemple (25), le premier tiret est celui qui figure à la fin d'une ligne, tandis que le second est difficile à justifier, étant donné que les deux phrases s'enchaînent du point de vue logique et narratif sans rupture :

(25) Le désir est sans doute très_louable de sa part, dans le poste où il se trouve actuellement; çependant, comme le rapport des affaires appartient proprement au Sécrétaire d'état, et que Mr. T., avec ses grands talens, que tout le monde connoit et admire, ne peut pas encore avoir çette habitude des grandes affaires qui ne s'acquiert que par l'expérience, il serait peut-être à souhaiter, qu'il ne fût pas appellé tout seul, mais suivi de M. R. _ C’est aussi çe qu'il souhaite lui-même, à çe qu'il assure : la seule çhose que je ne comprends pas ç'est pourquoi il n'a pas trouvé bon d'exprimer çe souhait, d'une manière plus intélligible, dans la lettre à l'Empereur, [...] (Ehrenström à Walleen, 1815).

Dans l'exemple (26), les phrases qui se trouvent des deux côtés du tiret constituent également les éléments d'une seule chaîne ou séquence, dont le sujet de conversation est la situation de la France lors des Cent-Jours. C'est le sujet qui occupe plus de la moitié de la lettre (et de plusieurs autres lettres d'Ehrenström et de Walleen en 1815). Ici, également, l'apparente rupture causée par le tiret semble difficile à expliquer :

(26) Nous sommes assez instruits par l'exprimer, pour savoir, que le succès d'une revolution, devient le germe d'une autre, et nous ne pouvons douter que l'exclusion prononcée de la Dynastie de Bourbon du Trône de France, comme un acte légal, ébranleroit fortement la sûreté des autres Dynasties légitimes, et compromettroit par conséquent la tranquillité et le bonheur des Nations. __ Malgré la facilité qu'a eû Bonaparte de reprendre la couronne et de chasser Louis XVIII, malgré l'attachement que les François montrent maintenant pour cet usurpateur, je ne puis pas me persuader que cet attachement soit si général et si sincère, qu'on veut nous le faire croire ; (Ehrenström à Walleen, 1815).

\subsection{Le tiret dans les lettres d'Armfelt}

On peut dire que l'emploi du tiret est assez sobre et modéré chez les trois fonctionnaires, Ehrenström, Rehbinder et Walleen, même si sa fonction n'est pas toujours évidente. Par contre, Gustaf Mauritz Armfelt (cf. note 16) fait preuve d'une utilisation tout à fait différente de ce marqueur. Contrairement aux autres épistoliers, nous disposons de lettres de jeunesse d'Armfelt, datant des années 1780 (conservées aux Archives 
Nationales de Finlande, comme les autres correspondances) ${ }^{19}$. En plus des lettres officielles, il écrivait surtout à son père et à sa fiancée, qui deviendra son épouse :

(27) j'espere que ma petite Hedda se porte mieux de jour en jour et que la Madelaine pas encore repentante s'agrandie et s'embellie de jour en jour nous sommes a coup sur de retour au mi_ du mois prochain __ J'espere que ta santé sera alors assez retablie pour me reçevoir en bonne et chretienne femme _ si tant est que mes reves de cette nuit s'accomplissent; il ne faut pas penser a ces sortes de choses là à un camp oû il n'y a pas une seule femme mais il me sera impossible d'exister un instant sans le plus tendre attachement et l'amour le plus constant pour ma divine Hedda __ milles \& milles baisers de cœur \& d'ame a toi

Il s'agit ci-dessus de l'extrait d'une lettre qu'Armfelt, âgé de 31 ou 32 ans, travaillant au service du roi Gustave de Suède, envoie en 1789 à sa femme qui se trouve en Finlande. On voit qu'il n'y a presqu'aucun autre signe de ponctuation ; on trouve un point-virgule dans cet extrait, et dans le reste de la lettre, il y a quelques virgules, mais aucun point. Le tiret est donc le signe universel ou passe-partout pour séparer des énoncés. Ces lettres familières et familiales du jeune Armfelt représentent manifestement une oralisation de l'écrit, une irruption de l'oral dans l'écrit ( $c f$. Llamas-Pombo, 2017).

Autre exemple : cet extrait d'une longue lettre à son père, écrite à Stockholm à l'âge de 24 ans, en 1782, date à laquelle il était déjà au service du roi :

(28) Quand votre fullmagt ${ }^{20}$ sera pret je vous l'enverrois, on m'a dit qu'il coute beaucoup d'argent, c'est un malheureux pays oû on paÿe au poids de l'or le bonheur qu'on a de se sacrifier dans le service $\&$ de vivre dans la dependance toute sa vie parcequ'on a-pour avoir la malheureuse manie de n'emploÿer son bien qu'aux achats de places. Le baron de Cederhjelm le pere est certainement un homme de bien $\&$ util au paÿs oû il se trouve emploÿé mais son cher fils, quoique d'ailleurs fort honet garcon est retourné a Paris, attiré par sa Belle Deesse qui se ruine pour faire voÿager son cher Baron, je sens fort bien qu'on peut aimer, mais ma Belle ne me ferra jamais faire 3000 livre en perte, seulement pour la voir, mais ingen stans går så underligt till som här i verlden ${ }^{21}$

Je goute infiniment l'idee que vous avés d'aller vous etablir en garcon a Åbo. mad : Edner ne pourrat'elle pas faire votre cuisine Leijonhjelm est a la campagne ainsi je ne peux pas lui parler dela succession de Mylenfelz, mais je

\footnotetext{
${ }^{19}$ Armfelt est mort à l'âge de 57 ans, et il ne nous reste que peu de correspondance de lui avec les autres épistoliers présentés ici. Ehrenström et lui étaient très liés et on s'attendrait à ce qu'il existe une correspondance abondante entre ces deux gentilshommes. Peut-être ces lettres n'ont-elles pas été retrouvées.

${ }^{20}$ Pouvoir, procuration. (Italiques de J.H., comme dans la proposition traduite dans la note 21).

${ }^{21}$ " [...] nulle part les choses ne se passent d'une manière aussi bizarre que dans ce monde ». Après cette phrase en suédois, on passe à la page suivante.
} 
suis sur qu'on aura dela peine a trouver quelqu'un qui paÿe beaucoup dans ce paÿs-ci parceque on a deja enlevé jusqu'aux enfants de 3 ans.

Dans cet extrait, il n'y a qu'un seul tiret, vers la fin : il s'agit manifestement d'un changement de sujet. Il y a également un espace blanc ( $c f$. infra, $\$ 4.4$ ) assez important vers le début, dont on peut dire qu'il correspond aussi à un changement de sujet. La proposition en suédois à la fin du premier paragraphe est un échantillon de l'alternance codique pratiquée par Armfelt ; souvent, les passages qui alternent dans les deux langues sont beaucoup plus longs, et dans certains cas, il paraît impossible de dire quelle est la langue principale ou la langue matrice d'une lettre.

L'exemple suivant constitue la fin d'une lettre adressée à son épouse. On voit que le signe de ponctuation par excellence est le tiret, même si le point et la virgule sont également utilisés. On ne trouve pas non plus de logique précise dans l'utilisation des majuscules et des minuscules.

(29) $[. .$.$] __ je conçois ma chere amie, que tu trouveras cruelle une privation$ pour laquelle tu n'es point née ___ mais quelques années d'oeconomie et l'habitude retabliront aisement, des pertes chimeriques __ le Rö̈ s'était chargé de $4000 \mathrm{RD}$. de dettes qui n'ont point été paÿé ___ il faudroit vendre des esfets pour les paÿer. je voudrais completter ma bibliotheque, enfin tout calcul fait je partirai de Stockholm avec 2 a 3000 RD. de dettes, mais je n'oublierai rien pour amasser, comme jusqu'ici je n'ai rien oublié pour dissiper __ Notre sejour est prolongé jusqu'au dimanche _ ainsi ce ne sera que 24 heures plus tard que nous nous verrons _ Adieu ma bien aimable et bien chere Amie croÿés qu'après la felicité de disposer de moi, celle de meriter de te posseder sera le but de mes souhaits _ Oui ! ma tendre et bonne Amie __ n'en doutés point _ je serai digne un jour d'etre uni a la femme la plus vertueuse et la plus digne d'admiration et d'estime que je connaisse _ \& c'est alors que tu n'auras plus le courage de me faire entre voir ton desir de mourir ___ (Lettre datée (en suédois) du Jeudi saint, sans indication d'année, mais une autre main a marqué 1788 sur la première page)

Parmi les lettres d'Armfelt à des personnalités importantes, il y en a qui sont destinées au " chancelier du royaume » (rikskansler), terme qui désigne le précurseur $\mathrm{du}$ ministre des affaires étrangères. Le ton change très nettement dans ces lettres sous plusieurs aspects ; l'humeur ludique cède le pas à une attitude sérieuse, et l'aspect graphique des lettres est également très différent.

Voici une lettre écrite à 26-27 ans, où Armfelt fait un rapport sur les voyages du roi (abrégé S.M.) et du dauphin, le futur Gustave IV Adolphe (abrégé S.A.R.), dont il est le compagnon de voyage :

(30) Monseigneur!

Avec une santé assez foible, je commençai ma Garde chèz S.A. Royale mardi passé, je l'ai trouvé sage, obéissant, singulierement raisonable et sourtout très 
disposé à plaire aux personnes qui l'entourent, il n'y a pas eu entre nous ni dispute ni contradiction pendant tout le temps de ma Garde.

S. M. fit un voyage à Svartsjö mércrèdi au soir et le Prince fut de la partie, nous y restames jusqu'à vendredi, et S.A.R. eut le talent d'amuser le Roy souvent des heures entieres _ Notre départ d'ici est surement remis au 27 ou 28, car le Roy ne partira guère avant le 30 de ce mois ci pour Carlscrone. Le tems qui a bien voulu se mettre au beau nous est extremement favorable eû égard à nos promenades, nous emploÿons quatre à cinq heures de la journée à ce plaisir fortifiant et le Prince en parait au reste on ne peut pas plus content. Il y a quelques jours que S.M. m'ordonna de dire à DeRoches de venir chéz S.A.R. pour lui donner des leçons dans la langue française, mais comme Jacob a fort bien rempli cette tache, et que d'ailleurs il etoit difficile pour moi de le congedier, j'ai différé de parler à DeRoches sous différents prétextes, jusqu'au moment que je trouvois d'en avertir $\mathrm{V}^{\text {re }}$ Exc.

Le peu d'envie qu'a temoigné le Prince à parler Français, a determiné S.M. a faire venir chez lui de tems en tems le petit Lambert qui est de son àge, et qui joint à l'accent le plus pur, la tournure la plus honête et infiniment de douceur, ils causent Français dès que nous nous éloignons un peu, mais tant que nous sommes trop près, S.A.R. lui parle par signe. Le Prince a reçu la lettre de votre Excell. hièr a diner, avec un plaisir infini, il m'a dit qu'il comptoit bientôt y faire reponse, mais je vois d'avance que le Roÿ de Cocagne dont il est fort occupé nous enlevera des moments qu'on pourroit consacrer au travail _ La plus parfaite tranquillité, une union rare et non interrompue caracterise cette année-ci la societé de Drottningsholm, et tout le monde forme des voeux pour la continuation d'une chose aussi rare. Je vous supplie de vouloir me permettre de vous assurer des sentimens de respect et d'attachement avec lesquels j'ai l'honneur d'etre de $\mathrm{V}^{\mathrm{re}}$ Exc.

le très humble et très obéissant serviteur

le Baron d'Armfelt (Lettre non datée ; ca. 1783/1784)

Le premier des deux tirets (vers le début du deuxième paragraphe) n'est pas facile à justifier ou à expliquer, tandis que le second précède une phrase que suivent les formules finales habituelles. La phrase suivant le tiret constitue une sorte de résumé du point de vue d'Armfelt sur la famille royale et leur vie et s'explique de cette façon-là.

Les lettres d'Armfelt se placent donc, d'après ces quelques exemples, sur une échelle allant d'une assez grande formalité, comme dans celles qui sont adressées au chancelier, aux lettres envoyées à ses proches, surtout à son épouse. Celles-ci font preuve d'une désinvolture aussi bien dans le respect de la grammaire que dans le style et surtout la ponctuation. Nous avons vu, en effet, que la ponctuation ordinaire disparaît presque totalement dans les lettres adressées à Hedvig (que son mari appelle de son petit nom Hedda ; $c f$. exemple 27) et qu'elle est remplacée par les tirets. Les correspondances d'Armfelt se démarquent de celles des trois autres épistoliers par la présence 
ostensible de l'axe diamésique et par une oralisation fréquente de l'écrit (LlamasPombo, 2017 : 51-52, 64, 75-77). Le code oral fait irruption dans le texte au moyen des signes de ponctuation.

La longueur des tirets varie beaucoup, bien qu'il soit évidemment difficile de la mesurer dans un texte écrit à la main, et a priori elle ne semble pas jouer de rôle dans les fonctions du tiret. L'espace pris par le tiret peut correspondre grosso modo à un seul caractère (comme c'est souvent le cas chez Rehbinder), mais aussi à plusieurs caractères.

\subsection{L'espace blanc}

La présentation du tiret dans les correspondances étudiées se justifie par la nouveauté de ce signe de ponctuation à la fin du XVIII ${ }^{e}$ et au début du XIX siècle, ce qui se voit dans le manque de codification chez les épistoliers. Malgré cela, on observe certaines similitudes entre les quatre fonctionnaires dans l'emploi de ce signe, même si les divergences sont peut-être plus importantes. Je voudrais terminer cet article avec une brève présentation d'un autre signe qui n'en est pas vraiment un (ou qui est en tout cas rarement considéré comme tel), à savoir, l'espace blanc, dont les correspondances des épistoliers finlandais témoignent toutefois d'une certaine existence. C'est surtout chez Ehrenström et Armfelt qu'on en trouve des exemples probants.

Il n'est pas facile de déterminer dans quel cas on peut soupçonner que l'utilisation de l'espace blanc est consciente et volontaire, et dans quel cas il s'agit simplement d'un geste fortuit. Souvent, les phrases consécutives forment des chaînes sans changement de topique, même s'il semble y avoir un espace blanc entre elles. C'est apparemment le cas chez Ehrenström ; l'espace blanc ne se rencontre pas dans toutes ses lettres, pas plus que le tiret :

(31) Bien de fois cependant j'ai été fortement tenté de vous entretenir sur les évenemens du jour, de rompre le silence que je m'étois imposé ; mais l'influence du principe a été plus forte que celle de mon penchant. J'ai pris une part sincère dans la perte que Vous venez de faire d'un Père cheri, en Vous félicitant en même tems du bonheur dont Vous avez joui, de voir sa vie prolongée jusqu'à un âge si avancé (Ehrenström à Walleen, 1818).

(32) [début de paragraphe] Encore une fausse allarme sur l'arrivée du Grand Duc. Cependant le General d'Opperman en a écrit officiellement à Sveabourg et a Abo. Le $\mathrm{B}^{\text {on }}$. de Rhebinder m'en a donné à cet égard les mêmes notions qu'il Vous a communiqué. Le Gouv : General qui attendoit ce Prince, et qui sans cela devoit être ici aujourd'hui, a remis son voyage à Sarila, et vient d'en entreprendre un à Björnebourg [fin de paragraphe] (Ehrenström à Walleen, 1819).

(33) On sait /maintenant $/ 22$ que le $\mathrm{B}^{\text {on }}$ de Rhebinder sera nommé Ministre d'Etat pour les Affaires de la Finlande; que Vous serez après lui, Secretaire d'Etat, et que $M^{r}$. de Haartman est venû pour s'arranger avec Vous, afin

\footnotetext{
${ }^{22}$ Mot ajouté au-dessus de la ligne.
} 
d'obtenir Votre Gouvernement. Ceci n'est pas un poisson d'Avril que je Vous envoie ; C'est un fait, que Votre confrere, le Gouverneur de ma Province, a réussi de pénétrer ce profond mystère, et a communiqué cette découverte, pendant son dernier séjour ici, pas à moi, mais à l'Amiral de Crit. Vous voyez que rien ne peut échapper à la perspicacité de certaines gens (Ehrenström à Walleen, 1819).

Dans (31), il y a effectivement un changement de topique, tandis que (32) et (33) illustrent la situation plus fréquente, où les phrases se succèdent sans interruption. Dans (33), la dernière phrase constitue cependant une réflexion finale quelque peu sarcastique ou désabusée ${ }^{23}$ et la pause blanche précédente peut souligner le poids à donner à cette remarque (comme aurait pu le faire aussi un tiret).

Il en va tout à fait différemment dans la correspondance d'Armfelt, comme l'illustre l'extrait suivant, provenant d'une lettre à son père ${ }^{24}$ :

C'est enfin après une suite d'evenements désagreables que je puisse mon tres cher Papa me rapeller dans votre souvenir___ La maladie de mon Prince tres vive et tres delicate me mit sur les dents, et mefit tomber malade assés dangereusement, grace aux soins de Mons. Axel j'exsiste encore, la fievre dura continuellement 38 heures $\&$ apres cela j'eû un crachement de sang qui s'arrêta par 3 saignées[.] Enfin me voila retabli, aussi bien portant $\&$ brulant de desir de vous embrasser, mais cela ne se ferra qu'à lafin du mois de juin car actuellement leRö̈ m'enleve avec lui a Gripsholm, comme Capitaine de Gardes, quoique la Reine $\&$ le Prince restent ici en ville. J'ai des compliments a vous faire sur votre debut comme Gouverneur[.] Vous jouissez deja d'une reputation au dela de votre esperance et leRoÿ m'a dit qu'il etoit on ne peut pas plus content de vous, je vous garantis sa protection particuliere, mais en affaires de justice il n'est qu'un Zero $\&$ les tetes à peruque sont tous, $\&$ malheureusement dans cette classe vous n'avés pas trop d'amis j'ai lu votre memoire concernant l'affaire de Brehmer, [...]

Dans ce début de lettre, il y a trois espaces blancs assez nets, dont deux suivent un tiret. La première phrase de la lettre annonce ce qui est à venir, et les phrases qui suivent, précédées d'un tiret assez long et d'un blanc, explicitent de quoi il s'agit. Dans les deux cas suivants, il s'agit par contre d'un changement de topique. On peut constater qu'en comparaison des lettres de jeunesse débridées, relâchées et même farfelues écrites non seulement à sa femme, mais aussi à son père, celle-ci est tout à fait sérieuse. Cela démontre que quand il s'agit de parler affaires même avec des membres de la famille, le jeune Armfelt (ici âgé de 25 ans) sait observer un ton qui convient et surveiller également son français beaucoup mieux que dans les extraits que nous avons vus précédemment (lettres précédentes à son épouse et à son père ; ex. 27 à 29).

${ }^{23}$ En réalité, les prédictions du secret que rapporte Ehrenström ne se réaliseront pas.

${ }^{24}$ À noter le manque de la formule initiale de salutation, pratique assez courante chez Armfelt. 
Il y a lieu de faire encore une brève remarque sur les alinéas, qui se rapprochent formellement des espaces blancs. L'emploi des alinéas chez Ehrenström et ses collègues Rehbinder et Walleen n'a souvent rien de particulier et correspond plus ou moins à un emploi moderne. Parfois, cependant, ils se servent de paragraphes très longs, surtout quand il s'agit de discuter de questions épineuses comme la situation politique européenne en 1814-1815. Ehrenström et Walleen s'écrivent alors des lettres véhémentes dirigées contre Napoléon, la France et les Français en général, qui contiennent des paragraphes de plusieurs dizaines de lignes (même des pages entières) ( $c f$. note 5). Ces lettres pleines d'indignation et de fureur les rapprochent d'Armfelt, qui semble parfois oublier totalement l'emploi des alinéas même sur deux pages touffues. Le plus souvent, il les utilise néanmoins d'une manière assez régulière. Badiou-Monferran $(2017$ :150) parle de sous-alinéation dans ce cas-là, tandis que le concept de sur-alinéation s'applique aussi à certaines lettres, mais plus rarement, lorsque plusieurs paragraphes assez brefs se suivent. En fait, on peut considérer l'emploi du «tiret d'énumération ou de structuration de texte " ( $c f$. exemple 17) comme un exemple de sur-aliénation.

\section{Conclusion}

Il y a parfois, mais rarement, dans ces correspondances des tirets qui semblent être " ordinaires ", c'est-à-dire d'une longueur standard et en position normale. Il est cependant possible que ces tirets soient dus à la négligence des épistoliers, qui ne font pas non plus toujours attention à la différence entre accent aigu et accent grave, et dont les accents sont parfois des traits horizontaux. Les tirets en bas de ligne se maintiennent dans la correspondance en suédois durant le XIX ${ }^{\mathrm{e}}$ siècle; on en trouve nombre d'exemples ( $c f$. note 15). Comme il y a peu d'études sur ce sujet, il est difficile pour un non-scandinaviste d'en dire beaucoup. À ma connaissance, et sauf erreur, il n'y en a apparemment pas dans des documents écrits à la main en français ; les épistoliers présentés ici ont dû suivre l'exemple donné par les épistoliers suédois du XVIII siècle, et ils emploient donc le tiret en bas de ligne aussi bien dans les lettres en suédois qu'en français. Plusieurs facteurs qu'on retrouve dans le corpus que j'ai étudié indiquent que les épistoliers sont, dans l'écriture de leurs lettres, à la recherche d'un système ou d'un code, qui sera mis en place au cours du XIX ${ }^{\mathrm{e}}$ siècle. Il s'agit de :

(a) paragraphes très longs, ou parfois mal délimités (mise en page ; sous-alinéation) ; parfois cependant plusieurs paragraphes sont très courts ;

(b) l'emploi de certains signes, surtout du tiret en bas de ligne qui disparaîtra au XIX ${ }^{e}$ s., et du manque d'autres signes (les parenthèses et les points de suspension) ;

(c) espaces blancs qui fonctionnent aussi comme signes de ponctuation ou de démarcation (signes de décrochage énonciatif).

Pour décrire ces caractéristiques communes aux quatre épistoliers, on pourrait employer avec profit les termes de syntaxe lâche et de ponctuation lâche (cf. Llamas- 
Pombo, $\left.2017: 75-76^{25}\right)$. On peut parler d'un diasystème suprasegmental des épistoliers, malgré la variation qu'on retrouve chez Armfelt : variation ou variance intra-subjective (registres différents sur un axe diastratique ; Llamas-Pombo, 2017 : 46), mais également variation par rapport aux autres épistoliers. C'est Armfelt qui se distingue très nettement de ses compatriotes et collègues, même si on doit admettre que nous ne connaissons pas de lettres de jeunesse écrites par Ehrenström, Rehbinder et Walleen et nous ne savons pas s'ils en ont écrit en français à cette époque.

\section{RÉFÉRENCES BIBLIOGRAPHIQUES}

AHLBÄCK, Olav (1971) : Svenskan i Finland. Stockholm, Läromedelsförlagen.

Archives Nationales de Finlande. URL : https://arkisto.fi/

BADIOU-MONFERRAN, Claire (2017) : " "Ponctuation noire", "ponctuation blanche" et "contes bleus" : l'évolution du codage des discours directs dans La Barbe bleue de Perrault (1695-1905) ", in Gabriella Parussa, Maria Colombo Timelli \& Elena LlamasPombo (éds), Enregistrer la parole et écrire la langue dans la diachronie du français. Tübingen, Narr Francke Attempto Verlag, 147-166.

BARSI, Monica (2008) : "La punteggiatura in Francia. Il settecento. L'ottocento e il novecento ", in Bice Mortara Garavelli (éd.), Storia della punteggiatura in Europa. Roma et Bari, Laterza, 267-293.

BREDEL, Ursula (2011) : Interpunktion. Heidelberg, Universitätsverlag Winter.

CATACH, Nina (1980) : "La ponctuation ». Langue française, 45, 16-27.

CATACH, Nina (1994) : La ponctuation. Paris, Presses Universitaires de France, (Coll. Que sais-je ? 2818).

DAHL, Alva (2016) : Interpunktion. Om skiljetecken och textens nyanser. S.l. [Stockholm], Morfem.

DRILLON, Jacques (1991) : Traité de la ponctuation française. Paris, Gallimard.

GAETA, Livio (2008) : «La punteggiatura nelle lingue scandinave », in Bice Mortara Garavelli (éd.), Storia della punteggiatura in Europa. Roma et Bari, Laterza, 503-512.

HÄRMÄ, Juhani (2013) : "Stratégies évidentielles dans des lettres finlandaises du XIXe siècle ", in Coco Norén, Kerstin Jonasson, Henning Nølke \& Maria Svensson (éds), Modalité, évidentialité et autres friandises langagières. Mélanges offerts à Hans Kronning à l'occasion de ses soixante ans. Bern, Peter Lang, 171-182.

HÄRMÄ, Juhani (2015) : « Stratégies de politesse dans des lettres finlandaises écrites en français au XIX ${ }^{e}$ siècle », in Sigrún Ástríður Eiríksdóttir (éd.), Actes du $19^{\circ}$ Congrès des

${ }^{25}$ Ces termes, lancés par A. M. Badía Margarit et José Polo, renvoient à la différence entre paratactique et hypotactique (par ex. chez W. von Wartburg). 
Romanistes scandinaves. Reykjavik, 12-15 aồt 2014. URL: http://conference.hi.is/rom14/files/2015/08/JUHANIHARMA.pdf.

HÄRMÄ, Juhani (2020) : «Le français et le suédois dans les correspondances finlandaises des $18 \mathrm{e}$ et 19e siècles : contacts de langues ", in Andreas Dufter, Klaus Grübl \& Thomas Scharinger (éds), Des parlers d'oül à la francophonie. Contact, variation et changement linguistiques. Berlin, Mouton De Gruyter (Beihefte zur Zeitschrift für romanische Philologie 440), 209-227.

HOLMES, Philip \& Ian HINCHLIFFE (2013) : Swedish. A comprehensive grammar. New York, Routledge.

KORZEN, Iørn (2017) : "La punteggiatura in danese e la guerra della virgola ", in Angela Ferrari, Letizia Lala \& Filippo Pecorari (éds), L’interpunzione oggi (e ieri). L'italiano e altre lingue europee. Firenze, Franco Cesati Editore, 325-343.

LINDGREN, Klaus \& Anna-Riitta LINDGREN (2005) : «Språkbyten bland ståndspersonerna i storfurstendömet Finland ", in Gabriel Bladh \& Christer Kuvaja (éds), Dialog och särart. Helsingfors, Svenska litteratursällskapet i Finland, 256-319.

LLAMAS-POMBO, Elena (2017) : "Graphie et ponctuation du français médiéval. Système et variation ", in Gabriella Parussa, Maria Colombo Timelli \& Elena Llamas-Pombo (éds), Enregistrer la parole et écrire la langue dans la diachronie du français. Tübingen, Narr Francke Attempto Verlag, 41-89.

ÖSTMAN, Margareta \& Hans ÖSTMAN (2008) : Au Champ d'Apollon. Écrits d'expression française produits en Suède (1550-2006). Stockholm, Kungl. Vitterhets Historie och Antikvitets Akademien.

ÖSTMAN, Margareta (2012) : Glanures servant de suite à Au Champ d'Apollon. Écrits d'expression française produits en Suède (1550-2006). Stockholm, Stockholms universitet.

PÉTILLON, Sabine (2004) : «Parenthèse et tiret double : pour une polyphonie mouvante ». L'information grammaticale, 102, 46-50.

PETTERSSON, Gertrud (2005) : Svenska språket under sjuhundra år. En historia om svenskan och dess utforskande. Lund, Studentlitteratur.

RETTIG, Wolfgang (2006) : "Romanismen in nichtromanischen Sprachen : Gallizismen ", in Gerhard Ernst et al. (éds), Histoire linguistique de la Romania. Manuel international d'histoire linguistique de la Romania. Berlin et New York, De Gruyter, vol. 2, 18061821.

SAARI, Mirja (2012) : The development of Finnish into a national language, in Matthias Hüning, Ulrike Vogl \& Olivier Moliner (éds), Standard Languages and Multilingualism in European History. Amsterdam et Philadelphia, John Benjamins, 179-204.

SERÇA, Isabelle (2004) : « La ponctuation : petit tour d'horizon ». L'information grammaticale, $102,11-17$.

STRÖMQUIST, Siv (2013) : Skiljeteckensboken. Skiljetecken, skrivtecken och typografiska grepp. S.l. [Stockholm], Morfem. 
WOLF, Barbara (2008) : «Romanische Sprachen als Verkehrssprachen : Neuzeit », in Gerhard Ernst et al. (éds), Histoire linguistique de la Romania. Manuel international d'histoire linguistique de la Romania. Berlin et New York, De Gruyter, vol. 3, 3318-3339. 